\title{
Uso del monitor cardiaco implantable en fibrilación auricular
}

\section{Use of implantable cardiac monitors in atrial fibrillation}

\author{
Diego Ignacio Vanegas-Cadavid
}

Unidad de Electrofisiología Cardiovascular, Hospital Militar Central, Bogotá, Colombia

Recibido el 3 de octubre de 2016; aceptado el 6 de octubre de 2016

Disponible en Internet el 10 de noviembre de 2016

\section{Monitor cardiaco implantable en el diagnóstico de fibrilación auricular}

La fibrilación auricular es una arritmia que usualmente se presenta en forma de paroxismos aislados y/o recurrentes de intensidad variable, que puede cursar con o sin síntomas. Diversos estudios poblacionales han documentado que es la arritmia más frecuente en el ser humano, presente en aproximadamente un $1 \%$ de la población general y hasta en el $10 \%$ de individuos mayores de 80 años, siendo asintomática entre un $10-30 \%$ de la población afectada ${ }^{1}$. Sin embargo, la incidencia real de la fibrilación auricular asintomática y/o sintomática depende de varios factores; el más importante quizá se refiere al tipo de método y tiempo de monitorización electrocardiográfica utilizada para su documentación².

\section{Monitorización ideal para la detección de fibrilación auricular}

La documentación de una alteración electrocardiográfica coincidente o no con un síntoma, puede lograrse con diferentes métodos. En tal sentido, el electrocardiograma convencional es el más sencillo y es considerado estándar de oro, pero es el menos accesible en el momento de los síntomas. También se dispone de la monitorización con Holter de 24-48 horas hasta siete días y la monitorización externa

Correo electrónico: diegovanegascadavid@gmail.com «de asa» o aquella satelital continua que puede extenderse por 30 días; no obstante, dado que la fibrilación auricular es paroxística y errática en su presentación y puede cursar asintomática, estos tipos de monitorización con frecuencia pueden no lograr su captura. En contraste, el monitor cardiaco implantable es una forma de monitorización invasiva altamente sensible y específica para la detección de episodios de fibrilación auricular, que tiene sensibilidad y especificidad del $97 \%$ y el $85 \%$, respectivamente (XPECT Trial) $)^{3,4}$.

\section{Usos aceptados del monitor cardiaco implantable}

De acuerdo con las guías de recomendaciones de la monitorización electrocardiográfica ambulatoria de la Asociación Americana del Corazón (AHA) y del Colegio Americano de Cardiología (ACC), el monitor cardiaco implantable puede ser considerado como opción útil y efectiva con recomendación clase I en ${ }^{5,6}$ :

1) Pacientes con episodios de síncope, pre-síncope o mareos episódicos de causa no aclarada o cuya causa no es obvia (figura 1).

2) Pacientes con palpitaciones recurrentes inexplicadas.

Para la Sociedad Europea de Cardiología ${ }^{7}$ el uso del monitor cardiaco implantable es una recomendación clase IIA, nivel $B$ en relación con las palpitaciones: el monitor cardiaco implantable puede estar indicado en casos seleccionados de 

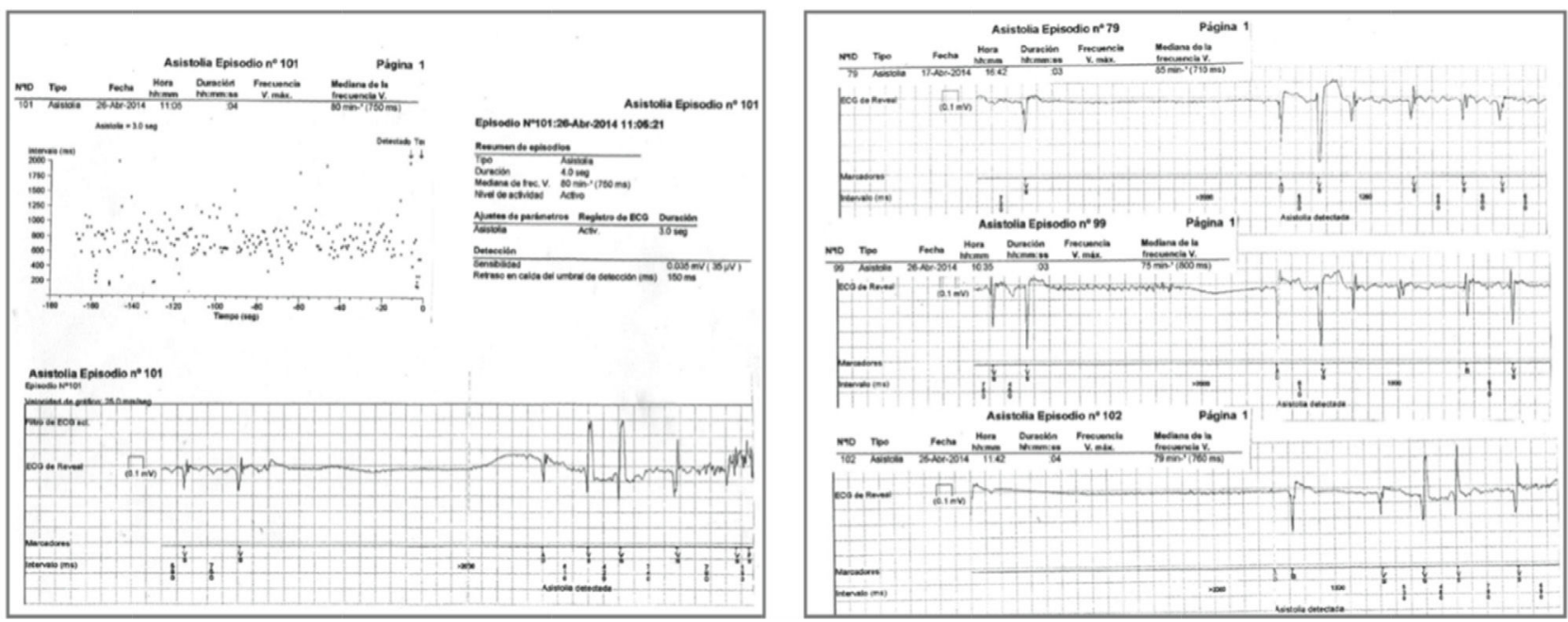

Figura 1 Mediante monitor cardiaco implantable se observan múltiples trazos de la detección de paroxismos de fibrilación auricular que terminan bruscamente en pausas mayores de 3 segundos, con escape sinusal y reinicio casi inmediato de la arritmia. Paciente de 72 años con síncope recurrente, episodio traumático, sin documentación electrocardiográfica de las pausas a pesar de ECG, mesa inclinada, Holter de 24 horas y prueba de esfuerzo. El diagnóstico se logró tras seis meses de monitoría invasiva. Un marcapaso definitivo fue la solución a su problema recurrente de síncope.

pacientes con palpitaciones sintomáticas infrecuentes pero de intensidad severa cuando un monitor cardiaco externo u otro sistema de monitorización electrocardiográfica ha fallado en documentar la causa subyacente ${ }^{7}$.

La fibrilación auricular (principalmente la forma paroxística de reciente inicio, sin excluir las otras formas de la arritmia) se manifiesta frecuentemente con palpitaciones recurrentes asociadas o no a otros síntomas (mareo, adinamia, dolor de pecho, síncope o presíncope). Aquellos pacientes con palpitaciones recurrentes sin documentación electrocardiográfica de una arritmia y bajo sospecha de fibrilación auricular no documentada, pueden ser considerados para el implante de un monitor cardiaco bajo la recomendación dirigida a desenmascarar o documentar la causa de las palpitaciones recurrentes inexplicadas.

\section{Potencial uso del monitor cardiaco en fibrilación auricular}

A. Para su diagnóstico o identificación:

1. Para su diagnóstico «de novo» en presencia de palpitaciones recurrentes.

2. En ataque cerebrovascular criptogénico, sospecha de fibrilación auricular como causa de embolia.

B. En fibrilación auricular conocida:

1. Establecer el carácter paroxístico y/o persistente (figura 2).

2. Evaluar la respuesta ventricular durante los episodios de fibrilación auricular.

3. Documentar patrón de inicio y terminación.

4. Establecer relación causa-efecto o circunstancias de aparición de los paroxismos (por ejemplo en relación con apnea del sueño, ejercicio, alcohol u otros hábitos)

5. Evaluar eficacia de tratamiento farmacológico $y / o$ aislamiento de venas pulmonares (figura 3).
6. Reconocer la coexistencia de otras arritmias relacionadas o no con intervenciones como el aislamiento de las venas pulmonares (flutter atípico, taquicardias auriculares incesantes)

7. Conocer carga y densidad arrítmica (figura 4).

\section{Importancia de la detección correcta y oportuna de la fibrilación auricular}

La importancia de la detección y cuantificación de los paroxismos de fibrilación auricular asintomáticos y/o sintomáticos, radica en su íntima relación con el ataque cerebro-vascular y/o la cardio-embolia, con sus secuelas correspondientes de invalidez o muerte. Esta arritmia es un factor de riesgo independiente para embolia cerebral con un incremento entre tres y seis veces. Así mismo, la recurrencia de la arritmia y el deterioro progresivo del ritmo cardiaco llevan a daño estructural y funcional de los atrios (miopatía atrial) y posteriormente de los ventrículos (taquicardiomiopatía), en cuyo caso la incidencia de falla cardiaca incrementa cuatro a seis veces. El impacto que esta arritmia tiene en los gastos estimados de los sistemas de salud es enorme (hasta 2,5\% de los gastos generales en salud en países desarrollados), de ahí que su detección correcta y oportuna debe facilitar consecuentemente su reducción al permitir su tratamiento adecuado y a tiempo ${ }^{8}$.

\section{Detección de fibrilación auricular y anticoagulación en ataque cerebro-vascular}

Por otra parte, la fibrilación auricular asintomática es tan ominosa o más que la fibrilación auricular sintomática en términos del daño estructural y funcional de las aurículas y en general del corazón, pero en especial de su potencial causa de embolia, dado que la arritmia no detectada no es tratada en forma adecuada (principalmente 


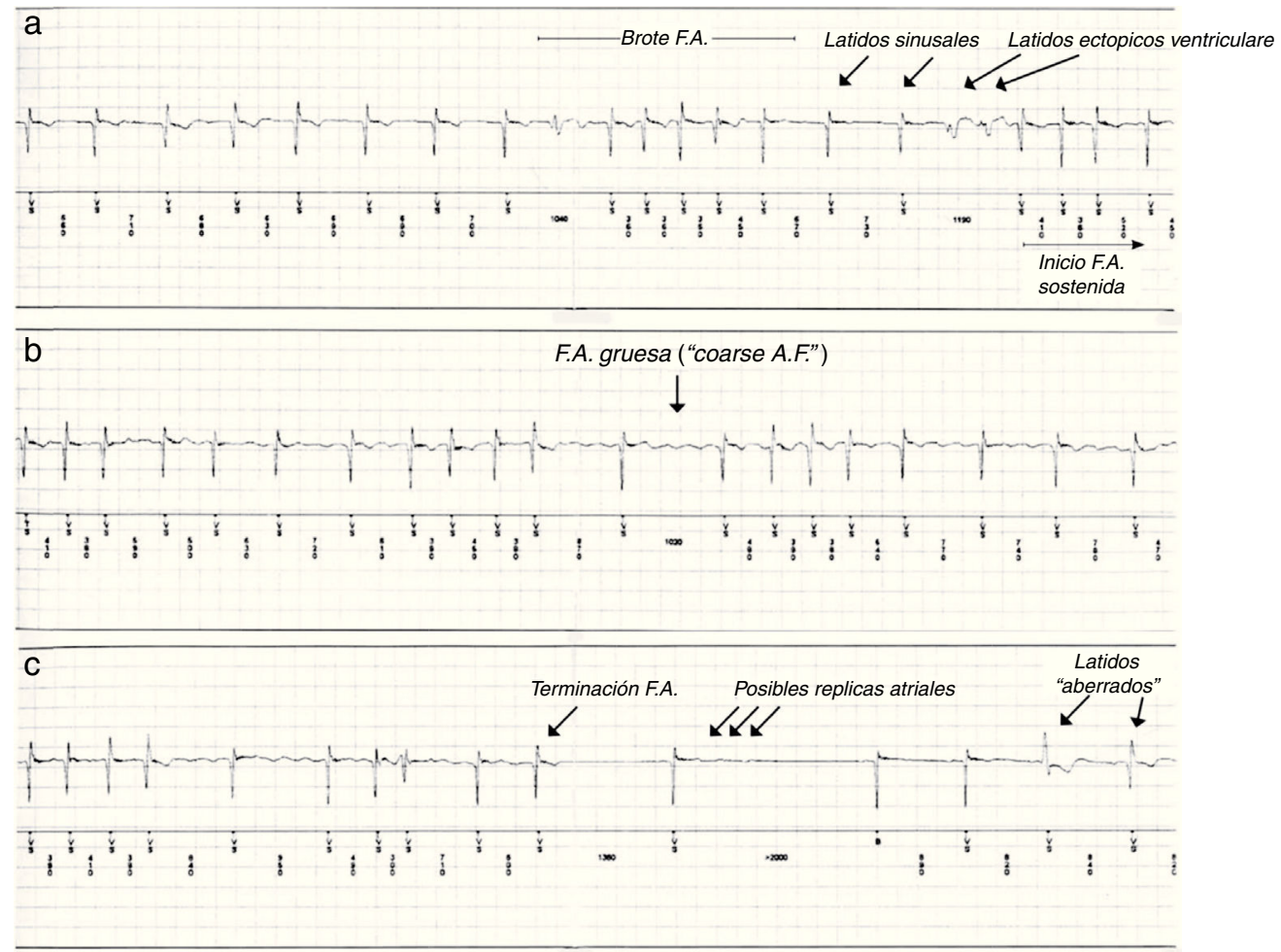

Figura 2 Episodio paroxístico de fibrilación auricular de 23 segundos de duración; los trazos a, b y c son continuos. Se aprecia el inicio y terminación, la mínima y máxima respuesta ventricular alcanzada, la forma de terminación y la aberrancia de conducción.

la estrategia de anticoagulación $)^{9,10}$. El ataque cerebrovascular cuya causa permanece desconocida (llamado por ello criptogénico), ocurre entre 20 a $40 \%$ de todos los eventos; la fibrilación auricular se reconoce como la causa más frecuente de embolia al sistema nervioso central y su incidencia ha sido detectada como creciente (8,9\%, $12,4 \%$ y $30 \%$ a 6,12 , y 36 meses respectivamente) cuando se practica monitorización cardiaca implantable a largo plazo ${ }^{6}$. El uso de anticoagulación en este grupo de pacientes con fibrilación auricular detectada mediante monitor cardiaco implantable, mostró una tendencia a la reducción de la recurrencia de nuevos eventos trombóticos en contraste con aquellos seguidos con monitorización convencional, posiblemente por el manejo oportuno $\mathrm{y} / \mathrm{o}$ profiláctico.

La fibrilación auricular también exhibe comportamientos que no son exclusivamente del carácter «blanco o negro», es decir que los episodios sintomáticos y asintomáticos coexisten en un mismo paciente. Se calcula que hasta un $50 \%$ de los episodios de fibrilación auricular postaislamiento de las venas pulmonares son asintomáticos y que el procedimiento es por sí mismo un factor de riesgo para fibrilación auricular asintomática, incrementándose su tasa de 1,1 preablación a 3,7 veces postprocedimiento ${ }^{11}$.

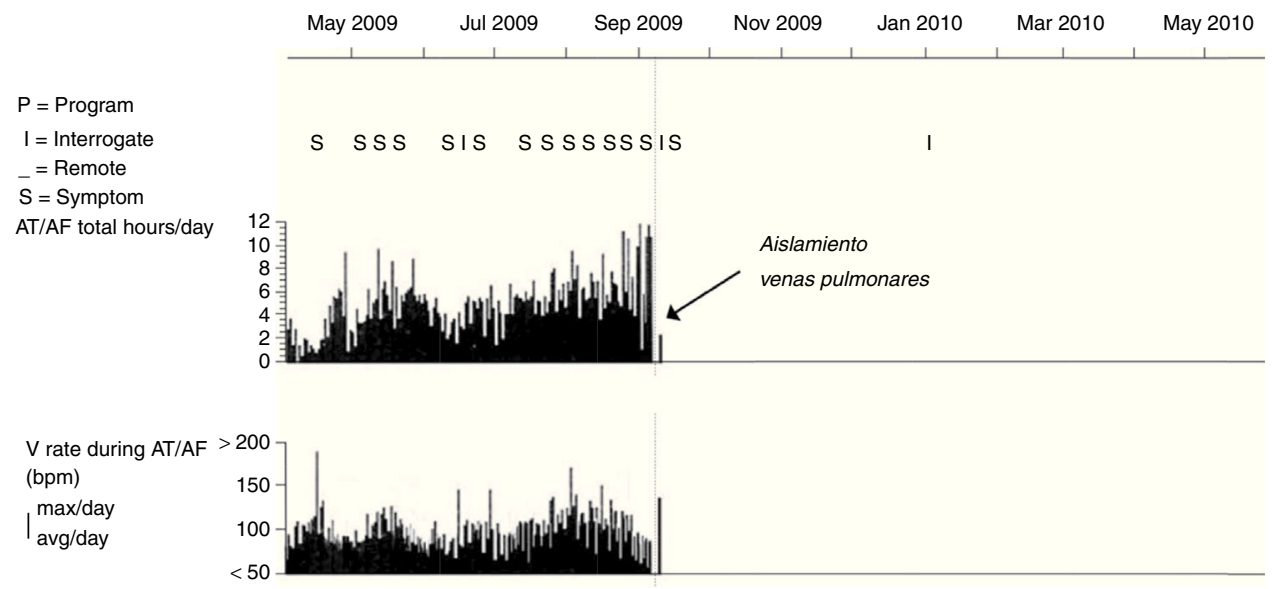

Figura 3 Registro de la carga de fibrilación auricular (alta) en un paciente de 68 años, con episodios diarios hasta de 12 horas de duración; tras ser llevado a aislamiento de las venas pulmonares hubo abolición de los registros de la arritmia. 
Dispositivo: Reveal XT 9.529

N. ${ }^{\circ}$ de serie: RAB667179S

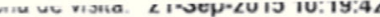

Sottware FullView SW007 Versión 7.1

Copyright $\odot$ Medtronic, Inc. 2010
Página 2

Tendencia de cardiac compass (Jul-2014 de Sep -2015)

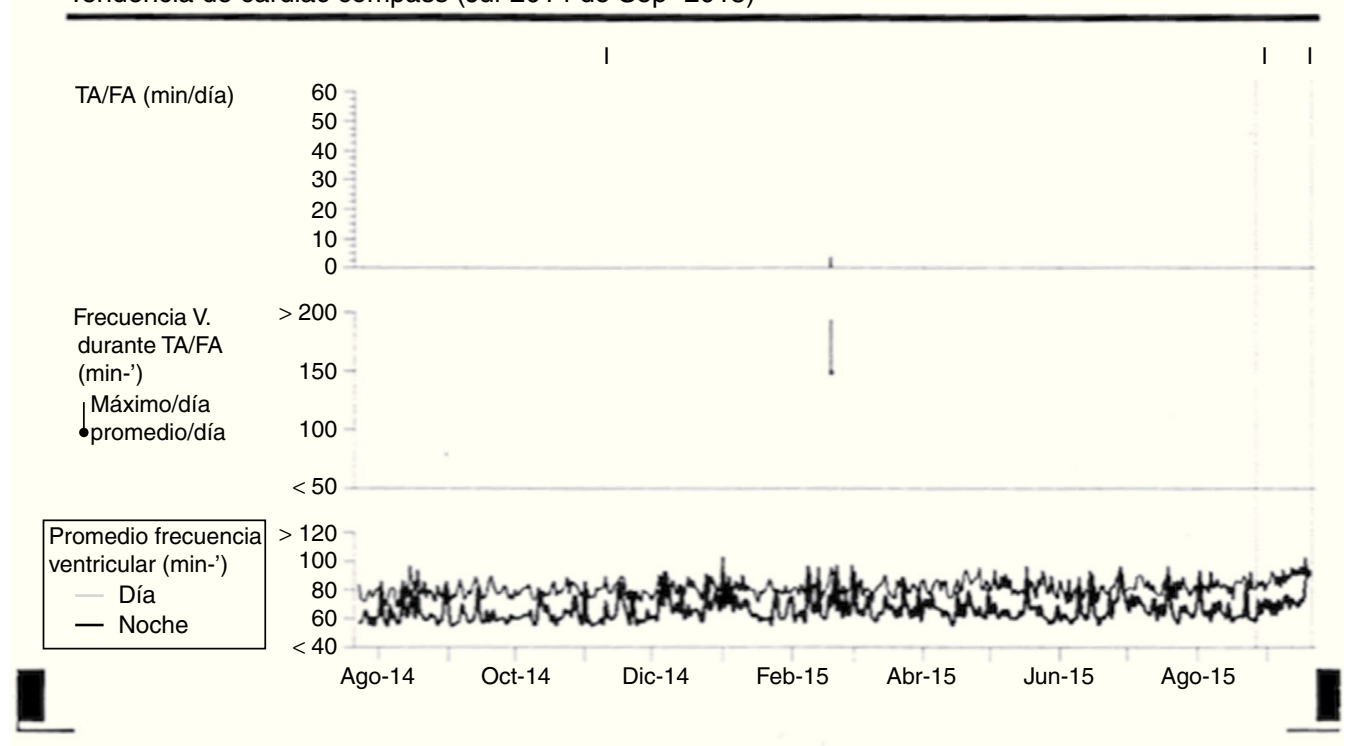

Figura 4 Registro de la carga de fibrilación auricular (baja) en un paciente con episodio de palpitaciones en quien se documentó esta arritmia en el servicio de urgencias. Tras autolimitarse sin tratamiento médico, recibió el implante de un monitor cardiaco con el fin de documentar recurrencia y definir potencial tratamiento farmacológico y/o de aislamiento de venas pulmonares. Durante un año de monitorización solo se documentó un episodio de fibrilación auricular de 4 minutos de duración.

\section{Detección de un patrón de fibrilación auricular}

La fibrilación auricular se cataloga como primer episodio, paroxística, persistente, largamente persistente y permanente, connotación que implica estrategias de manejo farmacológicas y no farmacológicas. Sin embargo, la determinación de ese carácter frecuentemente se basa en la clínica del paciente, principalmente el síntoma de las palpitaciones y su correlato electrocardiográfico. Se ha demostrado que la clasificación de la fibrilación auricular basada en la presunción clínica, puede errar al asignar un carácter paroxístico a pacientes en quienes se documenta mediante una monitorización más prolongada (utilizando el registro endocavitario de dispositivos implantables) una arritmia persistente, siendo posible también lo contrario ${ }^{12}$.

La fibrilación auricular tiene patrones muy irregulares que fluctúan en frecuencia y duración en el transcurso del tiempo, especialmente cuando se observa por largos periodos. Pacientes con carga arrítmica alta pueden experimentar fibrilación auricular paroxística y/o persistente; otros con carga arrítmica baja, comúnmente se catalogan con fibrilación auricular paroxística pero también pueden tener episodios de fibrilación auricular persistente. Dado que el monitor cardiaco implantable permite reconocer el episodio más corto y el más largo de fibrilación auricular, la carga de la arritmia, la frecuencia cardiaca durante los episodios, el patrón de inicio y la terminación, es razonable pensar que este dispositivo permitiría una objetivación de la dimensión de la arritmia y consecuentemente una aproximación terapéutica basada en una clasificación más realista

\section{Concepto de carga y densidad de la fibrilación auricular}

La fibrilación auricular puede cuantificarse utilizando el concepto de carga arrítmica, que determina el porcentaje o fracción del tiempo monitorizado en que el paciente se encuentra en fibrilación auricular. Así por ejemplo, una fracción o carga de 0,2 equivale a $20 \%$ del tiempo monitorizado en fibrilación auricular.

Un episodio de fibrilación auricular puede durar segundos, minutos, horas o días, por lo que el porcentaje del tiempo monitorizado en que el se documenta la arritmia, cuantificado en minutos al día (por ejemplo 20 minutos) puede hacer referencia a siete paroxismos o a uno solo. Así mismo, una carga arrítmica estimada en $20 \%$ de un tiempo determinado, por ejemplo un año, puede representar múltiples episodios cortos en duración pero muy frecuentes o quizá solo uno de larga duración persistente por varios días. La introducción del término «densidad arrítmica» se refiere a qupe tan «concentrados» o dispersos fueron los episodios o el tiempo que el paciente permaneció en fibrilación auricular. Una densidad alta significa que la agregación temporal de los episodios ocurrió en un tiempo determinado o que se presentaron concentrados o agrupados, mientras que una densidad baja significa dispersión de dichos episodios. Pacientes con cargas arrítmicas similares del $20 \%$ pueden tener, por lo tanto, densidades arrítmicas diferentes. Este concepto de la densidad arrítmica tiene connotaciones en relación con el tipo de monitorización empleado para detectar o clasificar la fibrilación auricular. Un ECG tres veces al año o una 
monitorización de Holter por siete días dos veces por año tomados al azar, pueden no detectar fibrilación auricular en un paciente con arritmia que exhibe densidad alta y carga baja (paciente que experimenta solo un episodio de fibrilación auricular por varios días consecutivos) mientras que esas estrategias pueden ser exitosas en la detección de episodios de fibrilación auricular de densidad baja y carga alta (paciente que experimenta brotes de fibrilación auricular cortos pero repetitivos y frecuentes).

\section{Monitor cardiaco implantable pre- y postaislamiento de las venas pulmonares}

La monitorización cardiaca implantable permite la documentación de la fibrilación auricular (carga y densidad arrítmica) antes de un aislamiento de venas pulmonares, y consecuentemente si esa documentación ocurre bajo tratamiento médico antiarrítmico puede demostrar la refractariedad 0 , por el contrario, su ausencia informa de la eficacia del manejo farmacológico; es la refractariedad o eficacia al tratamiento farmacológico una de las condiciones esenciales para que el paciente sea considerado candidato o no a ese procedimiento de acuerdo con las guías nacionales e internacionales. Posterior al procedimiento de aislamiento de las venas pulmonares, la documentación o no de la arritmia durante un tiempo prolongado de monitorización (por ejemplo tres a seis meses) puede demostrar qué paciente es respondedor o no a ese tratamiento al establecer el número y la frecuencia de los episodios ${ }^{12,13}$. Episodios aislados de muy corta duración y asintomáticos pueden llamar a una aproximación conservadora no intervencionista, mientras que una carga o densidad alta de paroxismos pueden indicar una nueva intervención.

Otros beneficios del monitor cardiaco implantable postaislamiento de las venas pulmonares son:

1. Hacer seguimiento de las potenciales recurrencias asintomáticas o sintomáticas.

2. Cuantificar la carga y densidad de los episodios posteriores al procedimiento.

3. Evaluar el tiempo de aparición entre el procedimiento y los primeros eventos detectados; si ocurren en el periodo «blanking» (tres meses posteriores al aislamiento) no se consideran predictores de fracaso, pero si están ausentes en las primeras dos semanas se considera predictor de éxito tardío ${ }^{12,13}$.

4. Determinar la potencial relación entre síntomas residuales o recurrentes y una arritmia específica.

5. Evaluar la incidencia de otras arritmias atriales como complicación del aislamiento de venas pulmonares, principalmente taquicardias auriculares y aleteos.

6. Confirmar la eliminación real de la fibrilación auricular y poder así definir estrategias de tratamiento farmacológico, principalmente antiarrítmicos y anticoagulación.

\section{Implicaciones futuras del uso de un monitor cardiaco implantable y de la anticoagulación}

De aparición muy reciente, se plantea la estrategia de anticoagulación guiada por la documentación de paroxismos de fibrilación auricular mediante un monitor cardiaco implantable con capacidad de transmisión inalámbrica y automática. En pacientes con fibrilación auricular paroxística, manejados farmacológicamente y/o mediante aislamiento de venas pulmonares con puntaje de $\mathrm{CHADS}_{2}$ de 1-2, el registro mediante monitorización invasiva y automática de episodios de fibrilación auricular con duración mayor a una hora permitió la administración puntual o específica de anticoagulantes orales por un tiempo limitado de 30 días en relación con el episodio detectado y no de manera permanente, lo que redundó en una reducción del tiempo bajo anticoagulación en un $94 \%^{13}$.

\section{Conclusiones}

El monitor cardiaco implantable es una herramienta muy útil para el diagnóstico «de novo» o en el seguimiento de la fibrilación auricular, en su cuantificación, registro de frecuencia, duración de episodios, carga y densidad arrítmica. El dispositivo ha demostrado alta sensibilidad y especificidad para el registro apropiado de esta arritmia, y la tecnología reciente permite su inserción de manera fácil con la enorme ventaja de una potencial monitorización inalámbrica. La monitorización cardiaca invasiva está proporcionando nueva información que ha permitido identificar con más certeza el papel de los episodios asintomáticos de fibrilación auricular, establecer la discordancia entre la clasificación clínica de la misma y la magnitud y frecuencia de los episodios documentados de la arritmia, así como facilitar el manejo y la aplicación de terapias individualizadas a la enorme variedad de escenarios clínicos resultantes de la heterogeneidad de esta patología.

\section{Conflicto de intereses}

Los autores declaran no tener conflicto de intereses.

\section{Bibliografía}

1. Sanna T, Diener HC, Passman RS, et al. for the CRYSTAL AF Investigators. Cryptogenic stroke and underlying atrial fibrillation. $\mathrm{N}$ Engl J Med. 2014;370:2478-86.

2. Hindricks G, Pokushalov E, Urban L, et al. Performance of a new leadless implantable cardiac monitor in detecting and quantifying atrial fibrillation. Results of the XPECT trial. Circ Arrhythm Electrophysiol. 2010;3:141-7.

3. Charitos El, Stierle U, Ziegler PD, et al. A comprehensive evaluation of rhythm monitoring strategies for the detection of atrial fibrillation recurrence: insights from 647 continuously monitored patients and implications for monitoring after therapeutic interventions. Circulation. 2012;126:806-14.

4. McLeod C, Gersh B. The epidemiology of atrial fibrillation. Chapter 1. In: Management of Atrial Fibrillation. A Practical Approach. Edited by: Shenasa M, Camm J. Oxford University Press. 2015. p. 1-2.

5. Mittal S, Movsowitz C, Steinberg J. Ambulatory External Electrocardiography. Focus on Atrial Fibrillation. J Am Coll Cardiol. 2011;58:1741-9.

6. Vanegas D. Monitor cardiaco implantable. Primera Edicion. Bogotá: Grupo Editorial Ibáñez; 2013. 
7. Marini C, DeSantis F, Sacco S, et al. Contribution of atrial fibrillation to incidence and outcome of ischemic stroke: results from a population-based study. Stroke. 2005;36:1115-9.

8. Arya A, Piorkowski C, Sommer P, et al. Clinical implications of various follow up strategies after catheter ablation of atrial fibrillation. PACE. 2007;30:458-62.

9. Klemm HU, Ventura R, Rostock T, et al. Correlation of symptoms to ECG diagnosis following atrial fibrillation ablation. J Cardiovasc Electrophysiol. 2006;17:146-50.

10. Charitos E, Pürerfellner H, Taya G, et al. Clinical classification of atrial fibrillation poorly reflects its temporal persistence. J Am Coll Cardiol. 2014;63:2840-8.

11. Oshi S, Choi AD, Kamath GS, et al. Prevalence, predictors, and prognosis of atrial fibrillation early after pulmonary vein isolation: findings from 3 months of continuous automatic
ECG loop recordings. J Cardiovasc Electrophysiol. 2009;20: 1089-94.

12. Pokushalov E, Romanov A, Corbucci G, et al. Does atrial fibrilation burden measured by continous monitoring during the blanking period predict the response to ablation at 12- month follow-up? Heart Rhythm. 2012;9:1375-9.

13. Passman R, Leong-Sit P, Adin-Christian A, et al. Targeted anticoagulation for atrial fibrillation guided by continous rhythm assessment with an insertable cardiac monitor: The Rhythm Evaluation for Anticoagulation With Continous Monitoring (REACT.COM) Pilot Study. J Cardiovasc Electrophysiol; 1-7. Disponible en: www.react.com. 